\title{
Evidence of semantic coding in short-term memory
}

H. C. A. DALE AND M, GREGORY

APPLIED PSYCHOLOGY RESEARCH UNIT, CAMBRIDGE, ENGLAND

An effect of semantic similarity in short-term memory was demonstrated and was compared with the effect of acoustic similarity. In free recall, using the $\mathrm{RI}$ paradigm, semantic similarity between $\mathrm{OL}$ and IL increased intrusions from IL, but decreased omissions. By contrast, acoustic similarity caused both IL - intrusions and omissions to increase.

The importance of acoustic similarity in short-term memory has been demonstrated in many experiments. Thus Conrad \& Hull (1964), Wickelgren (1965), and Baddeley (1966) have shown, using serial learning, that performance is adversely affected by inter-item acoustic similarity. Dale (1964) and Wickelgren (1965), have demonstrated a similar deleterious effect of acoustic similarity by using the RI paradigm and varying the similarity between OL and IL.

Attempts to demonstrate the effect of similarity along other dimensions in STM have been less successful, even though these are important in long-term memory. Baddeley (1966) varied acoustic-, semantic- and formalsimilarity in serial learning and obtained a substantial effect of acoustic similarity, a small $(6 \%)$ but statistically significant effect of semantic similarity, and no effect of formal similarity. Baddeley \& Dale (1966) found that whereas semantic similarity had a large RI effect in normal PA learning with lists of eight pairs being learned for eight trials, there was no such effect when the intra-list similarity of stimuli was varied in a minimal-PA learning task.

In view of these observations, the inference that shortterm memory uses an acoustic, or speech-motor, code would appear to be reasonable. The object of the present experiment was to challenge this conclusion with yet another attempt to demonstrate deleterious effects of semantic similarity in STM with the RI paradigm. Merhod

For each condition examined, two sets of six words were employed. For example, in the first replication the Acoustic, High Frequency, sets were: "blows, knows, rose, shows, flows, grows," and "dare, tear, pair, wear, share, care." On each trial, three words from one set, e.g., "rose, flows, shows," typed on a 3 in. $\mathrm{x} 4$ in. card, were shown to $S$ for 3.0 sec. during which time he was required to read them silently and memorize them (OL). The card was then turned over exposing six words which $\mathrm{S}$ had to read aloud (IL). In experimental trials, these were the three remaining words from the same set, each repeated once, e.g., "blows, grows, knows, grows, knows, blows." In control trials the three words were taken from the other set of the pair, e.g., "tear, pair, care, tear, care, pair."
Immediately after reading the IL words, each Sattempted to recall the original three words in order.

Trials were paced by a metronome set to a rate of 120 strokes per min. Two strokes were allowed for each OL word presented, two for turning over, and one for each IL word. Twenty-five sec. were allowed for each trial, which left $18 \mathrm{sec}$. for recall.

From each set of words, six triples were selected as stimulus words, three for experimental trials and three for control trials. Two balanced samples of material were produced to control any bias which could result from the use of particular triples as OL or IL.

Acoustic and semantic similarity was varied, together with word frequency, in a 2 by 2 factorial design. Fifty-seven of the 72 high frequency words were rated $\mathrm{AA}$ in the Thorndike Lorge count; the remaining 15 were rated $A$. All the low frequency words had a count of less than 50 per million, their mean value being 15.01 .

Each $S$ was given six experimental and six control trials with each combination of levels, making a total of 48 trials. Twelve Ss were given each sample of material; therefore a total of 24 was required per replication. There were three replications, each with fresh Ss and fresh material. The order of the 48 trials was randomized independently for each $\mathrm{S}$ by shuffling the cards.

\section{Results}

The overall results from all three replications are shown on Table 1, where each figure shows the total number of responses which fall within the indicated category.

Analysis of the total number of correct responses shows that performance was depressed to a significant extent by similar IL with both acoustic material $(p<0.001)$ and semantic material $(p<0.025)$. IL intrusions occurred significantly more frequently with similar distracting material in all conditions $(p<0.001$ in every case). In this respect the effect of semantic similarity was the same as that of acoustic similarity and it provides clear evidence that semantic coding is employed in at least one stage of the recall process under these conditions.

With omissions, there was an interaction between similarity and the form of coding being manipulated. With acoustic similarity there were more omissions when IL and OL were similar $(p<0.01)$. With semantic similarity there were significantly fewer omissions with similar IL $(\mathrm{p}<0.01)$.

Differences between replications have been ignored in the above analysis. These were generally small, but there was one significant exception: in the first repli- 
Table 1. Classification of Responses (1296 per column)

\begin{tabular}{|c|c|c|c|c|c|c|c|c|c|}
\hline \multirow{3}{*}{$\begin{array}{l}\text { Variable manipulate } \\
\text { Word Frequency } \\
\text { I.L. }\end{array}$} & & \multicolumn{4}{|c|}{ Acoustic Similarity } & \multicolumn{4}{|c|}{ Semantic Similarity } \\
\hline & & \multirow{2}{*}{\multicolumn{2}{|c|}{$\begin{array}{c}\text { High } \\
\text { Similar Different }\end{array}$}} & \multicolumn{2}{|c|}{ Low } & \multicolumn{2}{|c|}{ High } & \multicolumn{2}{|c|}{ Low } \\
\hline & & & & Similar & Different & Similar & Different & Similar & Different \\
\hline \multirow[t]{3}{*}{ Correct responses } & Total number & 668 & 904 & 610 & 765 & 909 & 940 & 830 & 866 \\
\hline & No. Correctly ordered & 492 & 655 & 398 & 487 & 725 & 806 & 661 & 699 \\
\hline & No. in Incorrect position & ก 176 & 249 & 212 & 278 & 184 & 134 & 169 & 167 \\
\hline \multirow[t]{4}{*}{ Intrusions } & Total & 287 & 88 & 293 & 220 & 226 & 134 & 224 & 114 \\
\hline & From OL set & & 36 & & 124 & & 71 & & 52 \\
\hline & From IL & & 113 & & 14 & & 31 & & 19 \\
\hline & Other & 43 & 39 & 40 & 82 & 22 & 32 & 29 & 43 \\
\hline \multicolumn{2}{|l|}{ Omissions } & 341 & 254 & 393 & 311 & 161 & 222 & 242 & 316 \\
\hline
\end{tabular}

cation, the semantic, high frequency, material yielded more omissions with similar distracting material than with different material. In this respect, this particular material behaved like acoustic material, with the result that there was an increased effect of similarity upon the overall performance level. The cause of this difference is not yet known.

\section{References}

Baddeley, A. D. Short-term memory for word sequences as a function of acoustic, semantic and formal similarity. Quart. J. exp. Psychol., in press.
Baddeley, A. D., \& Dale, H. C. A. The effect of semantic similarity on retroactive interference in long- and short-term memory. $J$. verbal Learn. verbal Behav., in press.

Conrad, R., \& Hull, A. J. Information, acoustic confusion, and memory span. Brit. J. Psychol, 1964, 55, 429-432.

Dale, H. C. A. Retroactive interference in short-term memory. Nature, 1964, 203, 1408 .

Wickelgren, W. A. Acoustic similarity and intrusion errors in shortterm memory. J. exp. Psychol., 1965, 70, 102-108.

Wickelgren, W. A. Acoustic similarity and retroactive interference in short-term memory. J. verbal Learn. verbal Behav., 1965, $4,53-61$. 